\title{
Cytokine intoxication as a model of cell apoptosis and predict of schizophrenia - like affective disorders
}

\section{Elena Viktorovna Drozdova*}

Department of Surgery, Samara State Medical University, Samara, Russia

For a long time there was no explanation of a study which had revealed that people with schizoaffective disorders and in particular suicidal attempts rarely get cancer. But now, we can assume that there are diseases that are "mirrored" because they occur with reverse/feedback pathophysiological mechanisms so that they are, in fact, antagonists.

That is obvious, for example, that hyperglycemia is an antagonist of hypoglycemia, that is why people with Alzheimer disease get cancer $50 \%$ more rarely and there are no data on morbidity of diabetes mellitus in people with Alzheimer's disease at all. These are extreme oppositions, since hypoglycemia can directly and indirectly, via reduction of serotonin release, steal pentose phosphate pathway and formation of lactate in neurons. Apparently, some processes act as a support. For instance: within the groups with daily consumption of protein and carbohydrates starting with group I - $16 \%$ protein and $49 \%$ carbohydrates, to group II $13 \%$ and $59 \%$ respectively, group III $-12 \%$ and $69 \%$, and IV group with $9 \%$ of protein and up to $74 \%$ of carbohydrate daily consumption, the coincidence rate of cancer and diabetes progresses significantly from group to group beginning with $40 \%$ within the first group, $52 \%$ in the second and $69 \%$ in the third group, and additional $38 \%$ in IV group ${ }^{*}[1,2]$

The Graph 1 displays coincidences of cancer and diabetes mellitus within the groups.

In this article we will consider the impact of serotonergic system and glucose level on cell proliferation and apoptosis. At first glance, stress-pathways and anti - apoptotic pathways go hand in hand: the higher level of CASPAS is, the more antiapoptotic pathways are suppressed and vice versa. Without any doubts, the excessive and one-time release of CASPAS causes overactivity of serotonergic system and stimulates powerful release of cytokines - up to cytokine storm and causes massive polymerization of death receptors. Moreover, simultaneous conflicting signals also cause a death of a cell.
More Information

${ }^{*}$ Address for Correspondence:

Dr. Elena Viktorovna Drozdova, Cardiovascular

Surgeon, Department of Surgery, Samara State Medical University, Samara, Russia Email: Elenadrozdova982@gmail.com; elenadrozdova.82@mail.ru

Submitted: November 25, 2021

Approved: December 16, 2021

Published: December 20, 2021

How to cite this article: Drozdova EV. Cytokine intoxication as a model of cell apoptosis and predict of schizophrenia - like affective disorders. Arch Asthma Allergy Immunol. 2021; 5: 038-040.

DOI: 10.29328/journal.aaai.1001028

ORCiD: orcid.org/0000-0002-9734-1412

Copyright License: (c 2021 Drozdova EV. This is an open access article distributed under the Creative Commons Attribution License, which permits unrestricted use, distribution, and reproduction in any medium, provided the original work is properly cited.

(D) Check for updates

(9) OPEN ACCESS

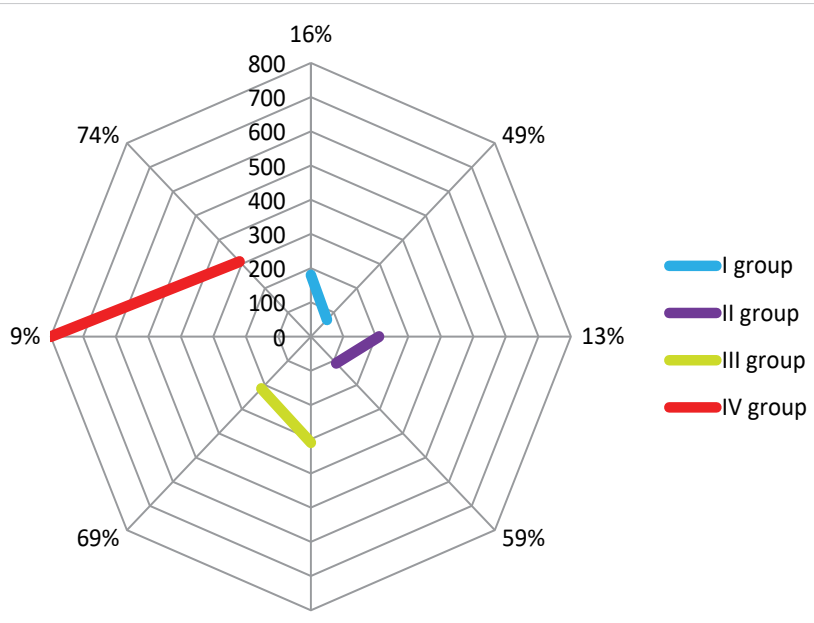

$12 \%$

Graph 1: Displays coincidences of cancer and diabetes mellitus within the groups.

That is the main way of pathophysiological realization of acute diseases and exacerbations of chronic inflammation and autoimmune diseases.

There are other factors that facilitate serotonin metabolism. First of all - hyperglycemia is one of the basic factors which triggers the serotonergic system in the direct way.

The next trigger for extra serotonin release is relative protein deficiency. Hypoproteinemia itself is a cause of violation of the control of serotonin synthesis and the 
decreased amounts of steroids and cholesterol potentiate the release of cortisol and speed it until the amount of cortisol is adequate for an immune response. This mechanism can occur with and without conditions of hyperglycemia.

Thus, both mechanisms increase the release of serotonin. Extraproduction of serotonin and consequently ACTH, GF and other glucose - dependant hormones leads to extraproduction of aldosterone and further to extraproduction of Ang2 and glucose. Hence, at this stage we can see how an organism makes up glucose resource if initially there was no hyperglycemia. Glucose has additional property: it doubles the amount of prorenin that in turn stimulates the release of rennin and tissue ANG2 which directly and indirectly activate rennin/ prorenin receptors [3] Graph 2.

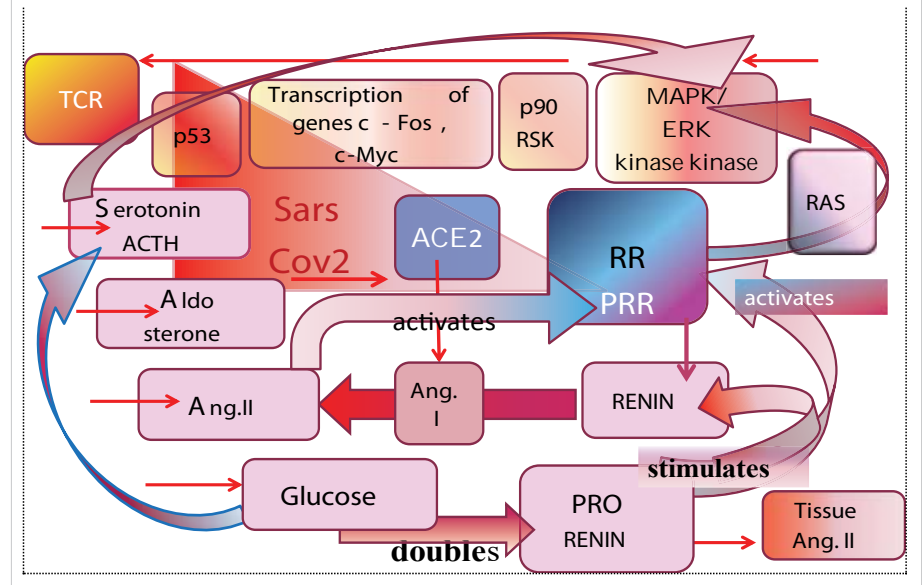

Graph 2: Displays the mutual influence of serotonergic and rennin angiotensin systems on triggering a cascade of both apoptotic and anti- apoptotic reactions. Starting with serotonin release and further causing release of aldosterone, Ang2 and glucose that increases the amount of prorenin and consequently activates rennin / prorenin receptors. The result is the quantitative and qualitative increase of rennin receptors. Moreover, activated rennin receptors can trigger MAPK cascade that in the presence of pathogen agents (like SARS-CoV2) can cause active cytokine reaction in both directions.

At this step we should ask the following question: whether this is a universe way to stimulate RAS that cause optimal conditions for activation of MAPKs, and what does it really mean? So, both stress-and anti -apoptotic ways ultimately mainly occur via MAPKs. With the in-depth study we can reveal some important specificities of the process. Firstly, there is a big temptation to assume that this is a cyclic process that is regulated by achieving the maximum quantitative level of regulatory proteins. In favor of this assumption HER2 can be given as an example. Overexpression of HER2/neu shifts the apoptotic - anti-apoptotic balance and upon reaching the maximal level masks the sensitivity to stress entirely; with the reduction of HER2 the activity of CASPAS increases. The same pattern shows PTEN that inhibits CASPAS activity via suppression of AKT activity, and consequently because of deactivation of GLUT4, which clearly supports the activity of serotonergic system exhibiting its diabetogenic property. The antagonist in this case is insulin that directly decreases glucose level and indirectly, via FOX proteins, deactivates AKT and consequently GLUT4 and changes the balance towards the stress pathway and apoptosis.

However, having looked at the structure of receptorsignaling proteins and domains we can see that Tyrosine residuals preferentially, if not always, bind with $\mathrm{SH} 2$ domain. Moreover, this pattern is typical for both cancer (including hemoblastosis) and inflammatory/autoimmune diseases. All the initial connections are set through the $\mathrm{SH} 2$ domains, and then add SH3 domains. That gives us the right to assume that originally the signal carries mitotic-proliferative information via $\mathrm{SH} 2$ adaptor. Thus, the main thing is, what exactly induces the switch of proliferation/maturation into apoptosis? In this case we can guess: this is due to joining SH3 domain that among other things is set by "stress-proteins" - Src - family, Irs, Grb2, STATs; while binding SH2-SH2, SH2-PTB, SH2PH occurs with "anti - apoptotic" proteins- Grb7, p38 and some specific nRTK like Syk, Zap70 that preferably lie in the pathophisiology of autoimmune diseases. PTB domain most likely functions as an insertion domain Irs- Tyr and also acts as an apoptotic signal transmitter. Also, it is logical to assume that if normally cell cycle arrest occurs at $G_{0}$, then extra cell deaths under the influence of CASPAS apparently are after $S$ phase.

If pain is an undeniable provoking factor of cell death, then reduction of sensitivity of hypotalamus due to increased osmolarity and consequently chronic dehydratation and ascites (in a way of SCLS) only presumably can be considered as an apoptotic signal and a reason of cancer decay. Besides, chronic dehydratation at $\mathrm{T} 4$ stage sometimes causes vein thromboses that are considered as cancer associated diseases.

This theory can explain why cytokines posses such diverse properties from hematopoesis to apoptosis and makes the plethora and pleiotropism of cytokines more explicable. COVID-19 pandemic highlighted that not only serotonin syndrome can cause respiratory syndrome, hypercoagulation, cardiomiopathy, neuropathy, irritable bowel syndrome and emotional instability. Most symptoms could be caused by cytokine intoxication, among them not only flu-like symptoms, but also lungs toxicity, oxygen desaturation, vomiting, nausea and also schizophrenia- like affective disorders. Moreover, we know that attempts of cytokine therapy caused predicted temporary side effects but also gave some unexpected results. For instance, increased amount of Il-8 or TNF $\alpha$ provoked the increased risk of schizophrenia in offspring. This theory acts as a support of virus oncology model just like famous Bittner virus. In this way cytokines serve as a direct mechanism of regulation of cell cycle and as a resource for innate immunization. Thus, after pandemic there is a high probability of the increase amount of newborns with the impaired serotonin metabolism and increased risks of cancer or schizo-affective disorders.

\section{Conclusion}

With the reduction of daily protein consumption from $16 \%$ 
to $9 \%$ and the increase daily carbohydrate consumption from $49 \%$ to $74 \%$ respectively, the coincidence rate of cancer and diabetes increseses significantly by $45 \%$.

Subclinical relative protein deficiency is a course of violation of the control of serotonin synthesis and acceleration of cortisol release that can occur with and without conditions of hyperglycemia.

Extraproduction of serotonin and consequently glucosedependant hormones leads to extraproduction of aldosterone and further to extraproduction of Ang2 and glucose.

Glucose can double the amount of prorenin that in turn stimulates the release of renin, tissue ANG2 which directly and indirectly activate rennin/prorenin receptors.

Both stress- and anti-apoptotic ways are set via MAPKs.
Originally, the signal carries mitotic-proliferative information via $\mathrm{SH} 2$ adaptor while joining $\mathrm{SH} 3$ domain causes the switch into apoptosis.

The clinical picture of the new coronavirus infection was largely determined by cytokine intoxication.

We can predict that newborns during and after pandemic might have impaired serotonin metabolism and increased risks of cancer or schizo-affective disorders.

\section{References}

1. 'Some Controversial Nutrition - Associated Aspects of Decompensation of Oncology and Diabetes mellitus. (Medical and Clinical Research ISSN:2577-8005)

2. The models of experimental oncology N A Popova.

3. Bioorganic Chemistry of Biological Signal Transduction Big Chemical Encyclopedia. 